\title{
SYSTEMIC INFLAMMATORY RESPONSE SYNDROME: THE CURRENT STATUS
}

\author{
Manisha Shrestha ${ }^{1}$, Anand Kumar ${ }^{2}$
}

\begin{abstract}
Systemic inflammatory response syndrome (SIRS) is a frequent and serious problem faced by clinicians in day to day practice and is a major factor of intensive care morbidity and mortality. The American College of Chest Physicians and the American Society of Critical Care Medicine in 1991 published definitions and criteria for systemic inflammatory response syndrome. Since then many researches have been undertaken to better understand the pathophysiology of systemic inflammatory response syndrome and to determine the accuracy of its diagnostic criteria. The criteria set by the 1991 consensus is still popularly used today. However, with the current knowledge on this matter many researchers have put forward the need of refinement in the criteria of systemic inflammatory response syndrome defined by 1991 consensus. This article aims to review the epidemiology, etiology, pathophysiology, laboratory diagnosis, treatment and the current views regarding SIRS.
\end{abstract}

KEYWORDS Systemic inflammatory response syndrome; SIRS; sepsis

1. Department of Pathology, Universal College of Medical Sciences, Bhairahawa, Nepal.

2. Department of Surgery, Universal College of Medical Sciences, Bhairahawa, Nepal

\author{
For Correspondence \\ Dr. Manisha Shrestha \\ Department of Pathology \\ Universal College of Medical Sciences \\ Bhairahawa, Nepal. \\ Email: mansha.stha@gmail.com
}




\section{INTRODUCTION}

Systemic inflammatory response syndrome popularly known as SIRS accounts for a major factor of intensive care morbidity and mortality. In earlier days, there was a lot of debate and confusion regarding the definition of sepsis, SIRS and multiorgan dysfunction syndrome (MODS) which necessitated the formulation of a conceptual and practical guideline. In 1991, the American College of Chest Physicians and the American Society of Critical Care Medicine published definitions and criteria for SIRS and sepsis. They set a diagnostic criterion for SIRS (Table 1) and described it as systemic inflammatory process irrespective of the cause and when SIRS was caused due to a confirmed infectious process, it was termed as sepsis.

\section{Table 1.Criteria for SIRS}

\begin{tabular}{|c|l|}
\hline Temperature & $>38^{\circ}$ or $<36^{\circ} \mathrm{C}$ (rectal temperature) \\
\hline Tachycardia & $>90$ beats $/$ minute \\
\hline Respiratory rate & $>20 /$ minute or $\mathrm{PaCO}_{2}<4.3 \mathrm{kPa}$ \\
\hline WBC count & $>12 \times 10^{9} / 1$ or $<4 \times 10^{9} / 1$ or $>10 \%$ immature forms (bands) \\
\hline \multicolumn{2}{|c}{ Presence of two or more of the above features qualifies for SIRS. } \\
\hline
\end{tabular}

This committee left the discussion open for refinement of the criteria after further research in the future, however the referred criteria is still equally popular but being debated in the present day. ${ }^{1}$

Many studies have been carried out since then to evaluate the prognostic and predictive accuracy of the criteria set by the 1991 consensus. These studies have led to arguments regarding the overly sensitive nature of the definition of SIRS, with most patients in the intensive care unit meeting the criteria, and lack of clinical specificity in the diagnostic criteria. ${ }^{2}$ This has led some researchers to put forward the need of a revised definition for SIRS. ${ }^{3}$

This article intends to review the epidemiology, etiology, pathophysiology, laboratory diagnosis, treatment and the current views regarding SIRS.

\section{EPIDEMIOLOGY}

The incidence of SIRS is high globally, although the exact incidence of it is not known due to the lack of specificity of its definition. One of the largest epidemiologic study on SIRS and sepsis carried out in the United states of America over a period of 22 years from 1979 to 2000 showed the average age of development of sepsis increasing over time with a mean increase of 3.5 years. It showed that sepsis was more common in males than in females and developed in later ages in females. ${ }^{4}$ A Brazilian study also showed males developed sepsis more than females and the respiratory tract was the most common source of infection. ${ }^{5}$ Study in Nepal had similar findings with sepsis being common in males and the most common source of infection was also the respiratory system.

The prevalence of SIRS was higher in intensive care unit patients and trauma patients had a higher risk of development of SIRS. ${ }^{7,8}$ The outcome of management was related to age, with poor outcome in elderly population, immune competence, associated co-morbidities, number of organs impaired, success of therapy and progression of disease to MODS in the individual. ${ }^{4,6,9}$

\section{ETIOLOGY}

It was initially presumed that only infectious conditions were responsible for the development of SIRS as the body's response to microbial products, such as 'pathogen-associated molecular patterns' (PAMPs). However, it did not explain the development of SIRS in patients with no infection and hence, theories held intestinal bacterial translocation as the cause of SIRS in these cases. ${ }^{10}$ It was only in the late 1980 s that researchers fully realized that SIRS could occur in the absence of an obvious source of infection. Subsequent studies revealed an endogenous 'damage- associated molecular pattern' (DAMPs) as the activator of the innate immunity and since then many other endogenous factors have been identified which contribute to the development of SIRS. ${ }^{11}$ The process of SIRS is initiated through an immune system trigger which includes infectious and non-infectious conditions, surgical procedures, trauma, drugs and therapies. ${ }^{12}$

\section{PATHOPHYSIOLOGY}

Irrespective of the etiology, SIRS follows a common final pathophysiology. Bone et $\mathrm{al}^{1}$ proposed three stages of SIRS. (Table 2)

\section{Table 2. Stages of SIRS ${ }^{1}$}

\begin{tabular}{|l|l|}
\hline Stage I & $\begin{array}{l}\text { Cytokines are released which promote wound repair and recruitment of } \\
\text { phagocytes directed at pathogen. }\end{array}$ \\
\hline Stage II & $\begin{array}{l}\text { Cytokines released in small amounts into the circulation to improve local } \\
\text { response whi ch increase the stimulation of growth factors and recruitment of } \\
\text { macrophages and platelets. Acute phase response is well regulated by a decrease } \\
\text { in pro-inflammatory mediators and attempts to restore homeostasis. }\end{array}$ \\
\hline Stage III & $\begin{array}{l}\text { If homeostasis is not restored, then massive system reaction occurs as a result of } \\
\text { autotoxic cytokines and MODS ensures. }\end{array}$ \\
\hline
\end{tabular}

These stages show SIRS to be a process of immune dysregulation where it is protective during stages I \& II and detrimental in stage III. ${ }^{1,13}$ There is another proposed theory i.e. CHAOS theory which proposes that SIRS ensues because of cardiovascular shock, lack of homeostasis, apoptosis, organ dysfunction and immune suppression.

SIRS represent a whole organism response to a variety of quite 
different immune challenge. The cells responsible are mononuclear leukocytes, platelets and polymorphonuclear leucocytes $(\mathrm{PMN})$ which release cytokines. These cytokines are soluble, low molecular weight glycoproteins which act as inflammatory mediators to regulate both innate and specific immune responses. At low concentration these cytokines have only paracrine effect but at higher concentration these have endocrine effect and act systemically. Tissue Necrosis Factor$\alpha$ (TNF) and Interleukin 1 (IL-1) are the first cytokines to be released within 1 hour of an insult and cause fever, release stress hormones and cleave the Nuclear Factor Kappa B (NF$\kappa \mathrm{B})$ inhibitor. After the inhibitor is removed, NF- $\kappa \mathrm{B}$ is able to initiate the production of mRNA that induce the production of other pro-inflammatory cytokines like Interleukins 6 (IL-6) and 8 (IL-8) and Interferon-gamma (IFN- $\gamma$ ). Cytokines especially IL-6, stimulate the release of acute phase reactants such as C-reactive protein. To counteract this proinflammatory state, the body is equipped with mechanism known as compensatory anti- inflammatory response syndrome (CARS) which includes Interleukin 4 (IL-4) and Interleukin 10 (IL-10) which are responsible for decreasing the production of TNF, IL-1, IL-6 and IL-8. The acute phase response also produces antagonists to TNF and IL-1 receptors. The balance of pro- inflammatory mechanisms and CARS is a critical factor in determining patient outcome. The pathogenesis of SIRS is illustrated in the flow chart. (Figure1)

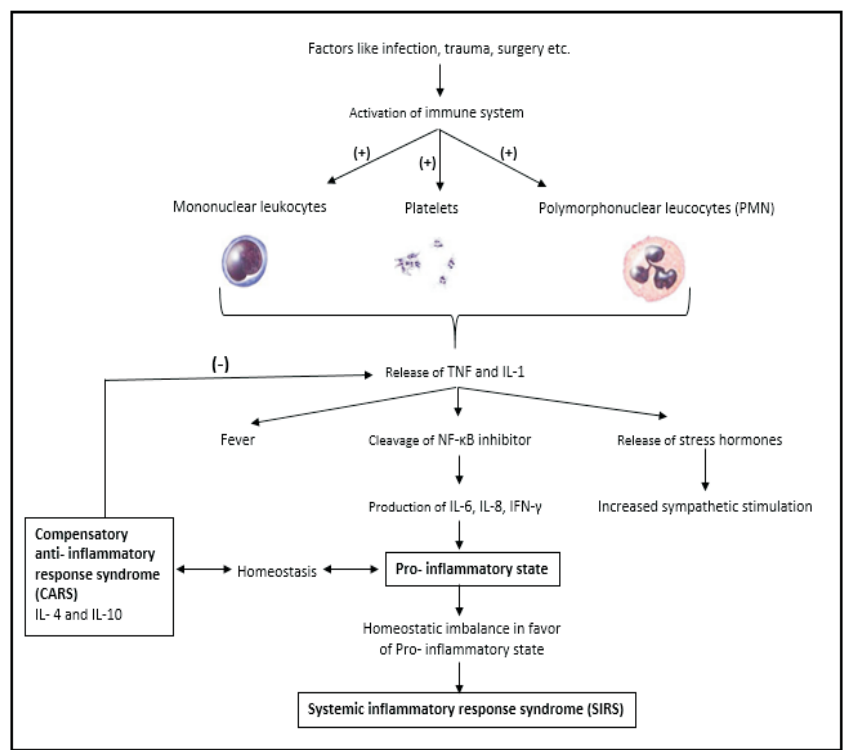

Figure 1. Pathophysiology of SIRS showing homeostatic imbalance in favor of pro- inflammatory state leading to the development of SIRS

Shift of this balance in favor of pro- inflammatory components will have a deleterious effect for the host and leads to
- Direct injury to cells

- Indirect injury through activation of serum factors, complements, Kinins, Kallikrein, coagulation and fibrinolysis cascades

- Endothelial injury and dysfunction which worsens inflammation through arachidonic acid metabolites, humoral and cell mediated immune mechanism.

The changes involved in the process are basically responsible for uncontrolled systemic vasodilatation, decreased peripheral vascular resistance and hypotension, poor tissue perfusion, third space loss, lactic acidosis and hemodynamic instability which may be refractory to volume and inotropic support. There is rise in energy demands, increased oxygen consumption, increased carbon dioxide production with metabolic acidosis, increased gluconeogenesis, lipolysis and aerobic glycolysis which are responsible for severe catabolic state and refractory to exogenous protein supplementation. Thus pro- inflammatory state, is responsible for tachycardia, tachypnoea, hyperpyrexia or hypothermia, hypotension, oliguria, leucocytosis / leucopenia, narrowed pulse and increased minute ventilation which are included in the diagnostic criteria for SIRS. ${ }^{1,12-14}$

\section{DIAGNOSIS}

The diagnosis of SIRS aims at evaluation of the possible causes which trigger the immune system. The investigations recommended are:

a. Biochemical/Hematological:

- Complete blood count - Leucocytosis / Leucopenia and $>10 \%$ immature cells (Bands)

- $\mathrm{ABG}$ Elevated lactate levels suggestive of metabolic acidosis and decreased $\mathrm{pH}$

- BUN / Creatinine ratio

- Increased transaminase, D Dimer studies, Prothrombin time / activated partial thromboplastic time (PT/aPTT) indicate towards haemostatic and hepatic dysfunction

- Serum albumin, calcium, magnesium, phosphate estimation

- C-reactive protein

b. Imaging studies: No specific imaging modality is recommended and its use depends on the suspected etiology.

c. Body fluids culture: Blood, urine, sputum, cerebrospinal 
fluid, cavity drainage fluids need to be cultured as per specific indications. ${ }^{12,1417}$

d. Cytokine assay: The important cytokines measured in the laboratory are TNF $\alpha$, IL-6 \& IL-8. Serum procalcitonin level is considered as a specific marker for SIRS. The levels of procalcitonin identifies the progression of SIRS to sepsis and MODS. ${ }^{1820}$ (Table 3 )

Table 3. Values of procalcitonin and their diagnosis ${ }^{1820}$

\begin{tabular}{|l|l|}
\hline Value of Procalcitonin & Diagnosis \\
\hline $0.5-2 \mathrm{ng} / \mathrm{ml}$ & SIRS \\
\hline$>2 \mathrm{ng} / \mathrm{ml}$ & Sepsis \\
\hline$>100 \mathrm{ng} / \mathrm{ml}$ & MODS \\
\hline
\end{tabular}

Newer biomarkers are under study like Angiopoietin 1 and 2 (Ang-1, Ang-2), which are ligands of the Tie-2 receptor expressed by endothelial cells. In normal conditions, the level of Ang-1 is higher so the Ang-1 Tie-2 complex predominates. During inflammation, however, Ang-2 is released from endothelial cells, which shifts the equilibrium to Ang-2Tie-2. Because of this relationship, Ang-2 levels and the Ang-1/Ang2 ratio have been proposed as possible biomarkers of sepsis and SIRS. ${ }^{21}$

Other biomarker under trial is triggering receptor expression on myeloid cells 1 (TREM-1). It is a part of the immunoglobulin superfamily and is expressed on the surfaces of phagocytic cells, with enhanced expression occurring after exposure of cells to fungi and bacteria and is a potential means of separating noninfectious SIRS from sepsis. ${ }^{16,22}$ Some studies suggest hemophagocytosis as an early indicator of advanced SIRS so bone marrow examination may be helpful. ${ }^{23}$ Many other biomarkers are under trial but since SIRS is a heterogeneous process, the use of a combination of biomarkers is recommended over a single marker. ${ }^{15}$

\section{TREATMENT OF SIRS}

A continuum exists in the process of SIRS to MODS and death so the critical time for treatment i.e. "Window of opportunity" for targeted intervention and better outcome is immediately after development of SIRS and before commencement of end organ damage. Treatment of SIRS is a team approach which comprises of physician, surgeon, intensivist, immunologist, physiotherapist, dietician. The guidelines of treatment include medical and surgical care, maintenance of nutrition, prophylaxis for pulmonary embolism and deep vein thrombosis, rehabilitation and physiotherapy and prevention of complications, some of which are discussed below. ${ }^{1,12,24}$

\section{VOLUME RESUSCITATION}

The primary objective is to restore tissue perfusion by achieving euvolemia and ensuring global oxygen delivery. Global delivery: Consumption ratios for oxygen are approximately $4: 1$. Mortality is approximately $50 \%$, when the ratio approaches nearly $2: 1$. This is best monitored using a pulmonary artery catheter (PAC) which guides volume therapy and also assesses cardiac performances. ${ }^{12}$

Nature of fluid could vary from crystalloid to colloid and aggressive fluid resuscitation is required to correct perfusion deficit. The selection of fluid depends on the patients' existing colloid oncotic pressure, serum albumin and serum electrolytes. Albumin resuscitation may be preferred and literature supports this statement. ${ }^{1,17}$

The precaution to be taken while correcting fluid is not to induce hyperchloremic metabolic acidosis particularly when colloid solutions are used. Hyperchloremia has an added disadvantage of inducing serine protease dysfunction, pain and postoperative nausea. ${ }^{25}$

\section{OPERATIVE INTERVENTION}

Surgery is done in patients with etiology of surgical origin and the extent of surgery would depend on indication. Surgical procedure can range from surgical drainage to debridement or resection/ removal of the source of infection. The surgical treatment is always supplemented with nutritional support, appropriate antibiotic use, specific organ support and postoperative resuscitation. ${ }^{17,24}$

\section{NUTRITIONALSUPPORT}

The objective is to attain positive nitrogen balance because of associated hyper-catabolism. The daily recommended calories are $2535 \mathrm{Kcal} / \mathrm{kg} /$ day or more depending on degree of hyper-metabolism. Excessive carbohydrate is avoided because of higher risk of hepatic lipogenesis and excessive carbon dioxide production. Fish oil rich in omega 3 polyunsaturated fatty acids may reduce leucotrines and prostaglandin $\mathrm{E}_{2}$ production thereby reducing the release of IL-1 and TNF. Vitamins, minerals and trace elements need to be supplemented. A consultation with nutritionist may be required for its correction. Enteral alimentation is always desirable than parenteral alimentation. ${ }^{12,24}$

\section{MEDICATIONS}

Medication is restricted to prophylaxis required for anticipated complications. A few drugs are recommended which have some beneficial results. ${ }^{13,17,24}$

- Steroids which can block the synthesis of cytokines 
- Pentoxyphyllin - A phosphodiesterase inhibitor, which interrupts intracellular signaling and decrease PMN \& TNF activity

- Monoclonal anti $\operatorname{TNF}(\alpha)$

- Activated protein C

\section{COMPLICATIONS}

The complications associated with SIRS are related to progression of the disease to sepsis, septic shock and multiorgan dysfunction syndrome. The common complications are acute renal failure due to acute tubular necrosis, acute lung injury or ARDS, protein caloric malnutrition, coagulopathy with acute hepatic insufficiency, hyperglycemia due to hyper metabolism and insulin resistance, stress ulcer, hypoalbuminemia, hypocalcemia, hypomagnesemia, hypokalemia and hyperchloremic metabolic acidosis.

The prognosis depends on timely intervention and control of proinflammatory state. The compensatory mechanism referred to as CARS is limited up to stage II of SIRS and is considered reversible and progression to stage III is referred as MARS (Mixed autogonistic response syndrome) carries increased mortality due to organ failure. . $^{13,26}$

\section{SIRS: Current views}

The criteria of SIRS developed in the 1991 consensus was shown to be overly sensitive and lacked specificity. ${ }^{3}$ Although this definition has been widely used in practice, it has been criticized by many experts. Another joint consensus in 2001 recognized the limitations of this definition but failed to provide any changes to the criteria due to lack of supporting evidence. $^{27}$ A 2016 commission organized by national societies including the Society of Critical Care Medicine (SCCM) and the European Society of Intensive Care Medicine (ESICM) proposed a new definition of sepsis, termed Sepsis-3 as a life-threatening organ dysfunction caused by a dysregulated host response to infection. ${ }^{28,29}$ The new definition recommended abandoning the use of SIRS criteria. $^{30}$

The committee also compared traditional SIRS criteria to other methods, including the Logistic Organ Dysfunction System (LODS) and Sequential Organ Failure Assessment (SOFA) scoring and Quick Sequential Organ Failure Assessment scoring (qSOFA). The SOFA scoring, which includes clinical and laboratory parameters, was recommended to assess the severity of organ dysfunction in potentially septic patients. qSOFA is a modified version of SOFA and consists of only three components, i.e. respiratory rate $>/=22 /$ minute, change in mental status and systolic blood pressure $</=100 \mathrm{~mm} / \mathrm{Hg}$, each allocated one point and qSOFA score of $\geq 2$ points indicating organ dysfunction. ${ }^{30}$ This method was set as a contemporary to the SIRS criteria. Since then, several studies have been carried out which showed SOFA score to have superiority in prediction of in-hospital mortality. ${ }^{31}$ However, the prognostic accuracy for in-hospital mortality between qSOFA and SIRS is still a matter of debate with some studies favoring qSOFA while others favored SIRS. 32,33

\section{CONCLUSION}

Further trials and research into this topic is advised to determine the accuracy of various scoring systems and criterias before dissolving the SIRS criteria. In addition, many healthcare facilities are currently using the SIRS criteria as part of emergency room and ICU protocol and application of the new recommendations will require time and funding to facilitate modification of protocols and retraining of healthcare providers. Taking all this into consideration, rushing to discard the SIRS criteria may not be such a wise decision in the present day context till a consensus is achieved.

\section{REFERENCES}

1. Bone RC, Balk RA, Cerra FB, et al. Definitions for sepsis and organ failure and guidelines for the use of innovative therapies in sepsis. Chest 1992; 101: 16441655.

2. Vincent J-L, Opal SM, Marshall JC, et al. Sepsis definitions: time for change. Lancet (London, England) 2013;381: 7745.

3. Balk RA. Systemic inflammatory response syndrome ( SIRS ) Where did it come from and is it still relevant today? 2014; 5: 2026.

4. Martin GS, Mannino DM, Eaton S, et al. The Epidemiology of Sepsis in the United States from 1979 through 2000. N Engl J Med 2003;348: 15461554.

5. Silva E, Pedro M de A, Sogayar ACB, et al. Brazilian Sepsis Epidemiological Study (BASES study). Crit Care 2004; 8: R25160.

6. Lakhey, S Karki, B Shrestha, B Shakya, S Pandey SB. Sepsis: a private hospital experience in Nepal. J Inst Med 2006; 28: 1215.

7. Baluch A, Janoo A, Lam KIM, et al. Septic shock: Review and anesthetic considerations. Middle East J Anesthesiol 2007; 19 7186.

8. Brun-Buisson C. The epidemiology of the systemic inflammatory response. Intensive Care Med 2000; 26 Suppl 1: S6474.

9. Angus DC, Linde-Zwirble WT, Lidicker J, et al. Epidemiology of severe sepsis in the United States: analysis of incidence, outcome, and associated costs of care. Crit Care Med 2001; 29: 130310 . 
10. Gentile LF, Cuenca AG, Efron PA, et al. Persistent inflammation and immunosuppression: a common syndrome and new horizon for surgical intensive care. J Trauma Acute Care Surg 2012; 72: 1491501.

11. Gentile LF, Moldawer LL. DAMPs, PAMPs, and the origins of SIRS in bacterial sepsis. Shock 2013;39: 1134.

12. Marino PL. The ICU book. 4th ed. Baltimore: Williams \& Wilkins, 2014.

13. Davies MHP. Systemic inflammatory response syndrome. 1997; 920935.

14. Paterson RL, Webster NR. Sepsis and the systemic inflammatory response syndrome. J R Coll Surg Edinb 2000; 45: 17882 .

15. Dunne WM. Laboratory Diagnosis of Sepsis? No SIRS, Not Just Yet. J Clin Microbiol 2015; 53: 24049.

16. Henriquez-Camacho C, Losa J. Biomarkers for Sepsis. Biomed Res Int 2014; 2014: 16.

17. Horn KD. QJM Evolving strategies in the treatment of sepsis and systemic inflammatory response syndrome ( SIRS ). 1998; 265277.

18. Brunkhorst F, Wegscheider K, Forycki Z, et al. Procalcitonin for early diagnosis and differentiation of SIRS, sepsis, severe sepsis and septic shock. Crit Care 1999; 3: P095.

19. Castelli GP, Pognani C, Meisner M, et al. Procalcitonin and Creactive protein during systemic inflammatory response syndrome, sepsis and organ dysfunction. Crit Care 2004; 8: R23442.

20. Arkader R, Troster EJ, Lopes MR, et al. Procalcitonin does discriminate between sepsis and systemic inflammatory response syndrome. Arch Dis Child 2006; 91:11720.

21. Vassiliou AG, Mastora Z, Orfanos SE, et al. Elevated biomarkers of endothelial dysfunction/activation at ICU admission are associated with sepsis development. Cytokine 2014; 69: 240247.

22. Oku R, Oda S, Nakada T, et al. Differential pattern of cellsurface and soluble TREM-1 between sepsis and SIRS Cytokine 2013; 61: 112117.

23. Kuwata K, Yamada S, Kinuwaki E, et al. Peripheral Hemophagocytosis. Shock 2006; 25: 344350.

24. Dellinger RP, Levy MM, Carlet JM, et al. Surviving Sepsis Campaign: International guidelines for management of severe sepsis and septic shock: 2008. Crit Care Med 2008; 36: 296327.

25. Shaw AD, Raghunathan K, Peyerl FW, et al. Association between intravenous chloride load during resuscitation and inhospital mortality among patients with SIRS. Intensive Care Med 2014; 40: 18971905.
26. Sugita H, Kinoshita Y, Baba H. The duration of SIRS before organ failure is a significant prognostic factor of sepsis. Int J Emerg Med 2012; 5: 44

27. Levy MM, Fink MP, Marshall JC, et al. 2001 SCCM/ ESICM/ ACCP/ATS/SIS International Sepsis Definitions Conference. Crit Care Med 2003; 31: 12501256.

28. Marik PE, Taeb AM. SIRS, qSOFA and new sepsis definition. J Thorac Dis 2017; 9: 943945 .

29. Singer M, Deutschman CS, Seymour CW, et al. The Third International Consensus Definitions for Sepsis and Septic Shock (Sepsis-3). JAMA2016; 315: 80110.

30. Singer M, Deutschman CS, Seymour CW, et al. The Third International Consensus Definitions for Sepsis and Septic Shock (Sepsis-3). JAMA 2016;315: 801.

31. Raith EP, Udy AA, Bailey M, et al. Prognostic Accuracy of the SOFA Score, SIRS Criteria, and qSOFA Score for In-Hospital Mortality Among Adults With Suspected Infection Admitted to the Intensive Care Unit. JAMA 2017; 317: 290.

32. Williams JM, Greenslade JH, McKenzie J V., et al. Systemic Inflammatory Response Syndrome, Quick Sequential Organ Function Assessment, and Organ Dysfunction. Chest 2017; 151: 586596.

33. Giamarellos-Bourboulis EJ, Tsaganos T, Tsangaris I, et al. Validation of the new Sepsis-3 definitions: proposal for improvement in early risk identification. Clin Microbiol Infect 2017; 23: 104109 . 